\title{
Envelope Following-Based RF Transmitters Using Switching-Mode Power Amplifiers
}

\author{
Je-Kuan Jau, Student Member, IEEE, Yu-An Chen, Tzyy-Sheng Horng, Senior Member, IEEE, and Jian-Yu Li
}

\begin{abstract}
This research presents a high-efficiency linear radio frequency transmitter applying the envelope-following technique to a switching-mode power amplifier. The use of predistorted envelope and quadrature-modulated signals can linearize the nonlinear behavior with fairly high average efficiency. The experimental results show that the proposed transmitter can deliver a $1.9-\mathrm{GHz}$ CDMA2000 $1 \times$ signal with high adjacent channel power ratio, low error vector magnitude, and high power added efficiency over a wide range of modulated output power.
\end{abstract}

Index Terms-Envelope following, linear radio frequency (RF) transmitter, switching-mode power amplifier.

\section{INTRODUCTION}

M ODERN digital communication systems usually apply the time-varying envelope modulation schemes, such as quadrature phase shift keying (QPSK) and quadrature amplitude modulation (QAM), to meet more stringent spectrum efficiency standards. The high peak-to-average power ratio (PAPR) in these modulation schemes usually requires a highly linear power amplifier (PA) with a sacrifice in efficiency. The envelope-following techniques in [1] and [2] can improve the linear PA's average efficiency by amplitude-modulating the supply voltage at the rate of the envelope variation via a Class- $S$ modulator. This can let the supply voltage vary positively with the envelope level so as to save average dc power consumption.

The envelope-following technique proposed in this letter replaces the linear PA with a switching-mode PA to further enhance the efficiency. The switching-mode PA operates like a switch with a distinguishing feature that the voltage waveform across the transistor hardly overlaps the current waveform through the transistor. This way, the power dissipated at the transistor is relatively lower to achieve higher efficiency in comparison to the other high-efficiency PAs. In addition, a switching-mode PA, such as Class-E PA, has an inherently linear relation between supply voltage and RF-output amplitude and can be treated as an amplitude modulator for restoring the envelope through the supply voltage onto a phase-modulated carrier, as used in the envelope elimination and restoration (EER) transmitters [3], [4]. However, in the proposed technique, since both RF-input and

Manuscript received January 23, 2006; revised May 2, 2006. This work was supported in part by the Ministry of Education, Taiwan, R.O.C., under the Aim for the Top University Plan and by the National Science Council of Taiwan, R.O.C., under Grant 94-2213-E-110-023.

J.-K. Jau, Y.-A. Chen, and T.-S. Horng are with the Department of Electrical Engineering, National Sun Yat-Sen University, Kaohsiung 804, Taiwan, R.O.C. (e-mail: eastpeak@gmail.com; yachen@gmail.com; jason@ee.nsysu.edu.tw).

J.-Y. Li is with the Information and Communications Research Labs, Industrial Technology and Research Institute, Hsinchu 310, Taiwan, R.O.C. (e-mail: JianYu@itri.org.tw).

Digital Object Identifier 10.1109/LMWC.2006.879471

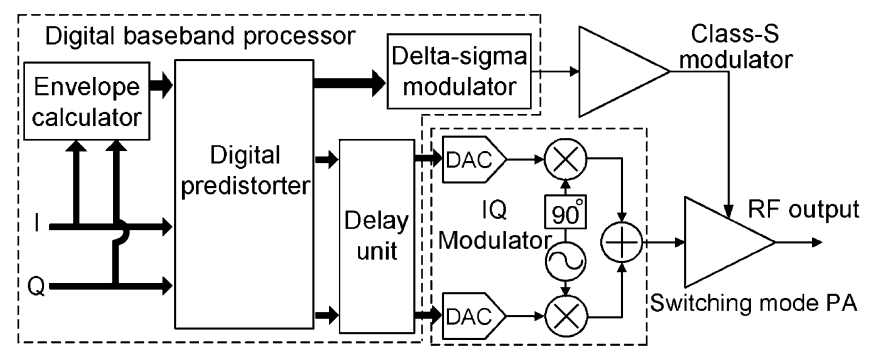

Fig. 1. Proposed envelope following-based RF transmitter using a switchingmode PA.

supply-voltage signals applied to a switching-mode PA contain the envelope variation, the RF-output modulated signal should be distorted due to the double amplitude modulation. Therefore, this research aims to work out a feasible solution for the envelope following-based RF transmitters that can use very highly efficient switching-mode PAs. After careful study, a digital predistorter designed for AM-AM compensation is proposed to improve the output signal distortion. As a result, a prototype transmitter for 1.9-GHz CDMA2000 1× applications has been implemented and proved to be highly linear and efficient based on the proposed technique.

\section{SYSTEM OVERVIEW AND IMPLEMENTATION}

The proposed transmitter system shown in Fig. 1 consists of a digital baseband processor, an $I Q$ modulator, a Class- $S$ modulator, and a switching-mode PA. The digital baseband processor mainly includes an envelope calculator, a predistorter, a delta-sigma modulator, and a delay unit. The envelope calculator aims to extract the envelope from the baseband pulseshaped $I Q$ signal in the digital domain. The predistorter is for compensating the above-mentioned AM-AM distortion due to the double amplitude modulation. The delta-sigma modulator is to replace the conventional pulse width modulator for driving the Class- $S$ modulator. The delay unit is required to compensate the phase mismatch between phase information and amplitude information at the combination point. The Class- $S$ and $I Q$ modulator takes charge of delivering an amplified analog envelope signal and a quadrature-modulated RF signal to be inserted into the supply-voltage and RF-input terminal, respectively, of the switching-mode PA.

\section{A. Class-E PA}

The Class-E PA classified as a switching-mode PA is quite popularly used in the EER transmitters because of its high efficiency and good linear relation between supply voltage and RF-output amplitude. Fig. 2(a) illustrates a Class-E PA 


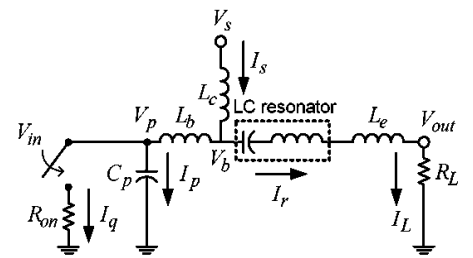

(a)

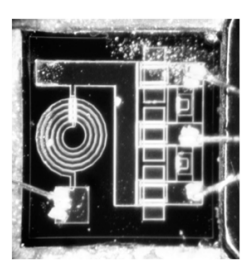

(b)
Fig. 2. (a) Equivalent circuit. (b) Chip micrograph of the designed Class-E PA.

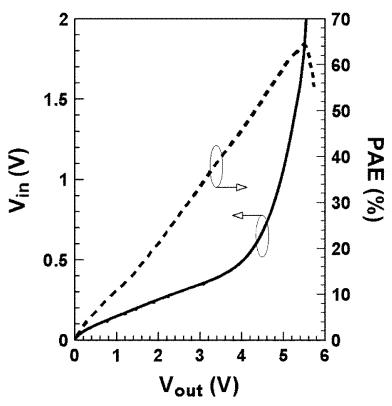

(a)

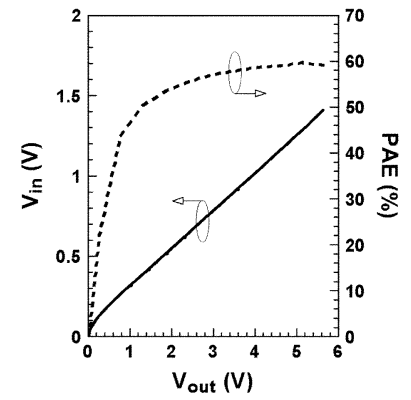

(b)
Fig. 3. RF input-output amplitude relation and PAE in the $\mathrm{CW}$ measurement of Class-E PA with (a) $V_{s}=3 \mathrm{~V}$ and (b) $V_{s}=2.33 V_{\text {in }}$.

design for the specific application to our proposed RF transmitter. To ensure high efficiency, the on-chip output shunt capacitance $\left(C_{p}\right)$ and off-chip output load-network elements in the Class-E PA must be carefully designed to eliminate as much waveform overlap as possible between the voltage $\left(V_{p}\right)$ across and the current $\left(I_{q}\right)$ through the power transistor. In addition, a small dc-feed inductance is used for increasing the modulation bandwidth at the supply-voltage terminal [5]. In [6], we have derived the closed-form design solutions for these output matching elements in simultaneous consideration of the switch-on resistance $\left(R_{\mathrm{on}}\right)$, dc-feed inductance $\left(L_{c}\right)$, and package bondwire inductance $\left(L_{b}\right)$.

According to the derived solutions, the Class-E PA was implemented in a $0.15-\mu \mathrm{m}$ InGaAs pHEMT process with a chip micrograph shown in Fig. 2(b), and then measured in continuous wave $(\mathrm{CW})$ at a fixed and variable supply voltage $\left(V_{s}\right)$ with results as shown in Fig. 3(a) and (b), respectively. It can be seen from Fig. 3(a) that at a fixed $V_{s}$ of $3 \mathrm{~V}$, the Class-E PA exhibits strong nonlinearity in the RF input-output amplitude relation with a power added efficiency (PAE) decreasing almost linearly from the peak value of $64 \%$ to a lower value as the RF-output amplitude is decreased. To linearize the Class-E PA, a variable supply voltage in proportion to the RF-input amplitude $\left(V_{\text {in }}\right)$ is applied. In Fig. 3(a), at $V_{s}=3 \mathrm{~V}$, the voltage gain is equal to $12 \mathrm{~dB}\left(G_{v}=3.98\right)$ for $V_{\text {in }}=1.29 \mathrm{~V}\left(V_{\text {out }}=5.13 \mathrm{~V}\right)$. Hence, the value of $V_{s} / V_{\text {in }}$ is 2.33 , which is chosen as the constant ratio to achieve the targeted 12-dB constant gain for all input amplitude levels up to $1.29 \mathrm{~V}$. From the measurement results shown in Fig. 3(b), such an amplitude-following supply voltage not only improves the linearity but also enhances the PAE quite noticeably for all output amplitude levels up to $5.13 \mathrm{~V}$.

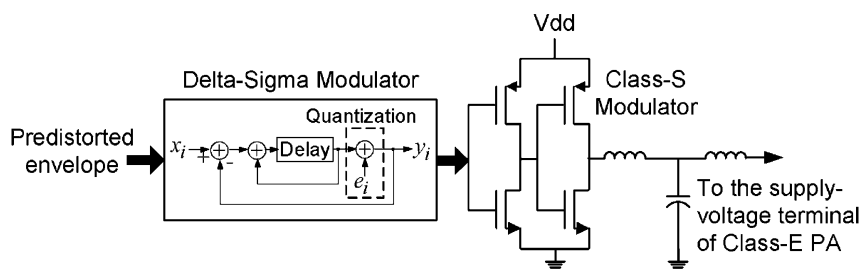

Fig. 4. Class- $S$ modulator driven with the delta-sigma modulator for highefficiency envelope processing.

\section{B. Digital Predistortion Scheme}

When the RF-input amplitude is large enough to switch the power transistor between saturation and cut-off, the Class-E PA can achieve a linear relation between RF-output amplitude and supply voltage with a high PAE up to 60\%, as shown in Fig. 3(b). However, at low RF-input amplitudes, the power transistor cannot switch on and off properly. Due to that, the Class-E PA reduces the gain and thus cannot maintain the linear $V_{\text {in }}-V_{\text {out }}$ relation anymore. In this research, a digital predistortion scheme is proposed to overcome this problem. The measured $V_{\text {in }}-V_{\text {out }}$ relation shown in Fig. 3(b) can be expressed as

$$
V_{\text {in }}\left(V_{\text {out }}\right)=\frac{V_{\text {out }}}{G_{v}}+\Delta V\left(V_{\text {out }}\right)
$$

where $\Delta V$ is the voltage error to account for the deviation from the linear part that corresponds to the targeted 12-dB constant gain. From (1), $\Delta V$ can be compensated for by designing a digital predistorter to modify $V_{\text {in }}$ as

$$
V_{\text {in }}^{\prime}=V_{\text {in }}+\Delta V\left(G_{v} V_{\text {in }}\right) .
$$

It is noted that $\Delta V$ is a smoothly varying function and can be curve-fitted as a polynomial for the purpose of implementing the predistorter as a digital arithmetic unit. In a digital modulation test, $V_{\text {in }}$ can be regarded as equal to the transient envelope of the applied quadrature-modulated signal.

\section{Envelope Processing Technique}

As shown in Fig. 4, a class- $S$ modulator driven with a firstorder delta-sigma modulator is used for processing the envelope signal highly efficiently. The delta-sigma modulator aims to transform an input multibit envelope signal into a binary one and its operation can be described in the $z$-transform domain as

$$
y=x z^{-1}+e\left(1-z^{-1}\right)
$$

where $x$ and $y$ are the input multi-bit signal and the output 1-b binary signal, respectively, and $e$ represents the quantization error. From (3), the output signal is regarded as unchanged except for a unit delay while the quantization error is differentiated. Due to the differentiation, the resulting quantization noise is pushed to high frequency. The Class- $S$ modulator consists of a CMOS buffer realized in a $0.35-\mu \mathrm{m}$ CMOS process to amplify the binary envelope signal, and a third-order equal-ripple Chebyshev low-pass filter as an output stage to further convert the binary envelope signal into an analog one with the filtered 
out quantization noise. The envelope processing efficiency is defined as the ratio of the average envelope output power to the dc consumed power, which is given as

$$
\eta_{e}=\frac{P_{\mathrm{env}}}{P_{\mathrm{dc}}}
$$

In the measurement, $\eta_{e}$ varies within $70 \%-80 \%$ in the applicable range of average envelope output power.

\section{Baseband Processor and IQ Modulator}

The baseband processor finally implemented using FPGA includes the pulse-shaping filter, half-band filter, interpolator, envelope calculator, predistorter, delay unit, and delta-sigma modulator. The pulse-shaping filter operates at four times the chip rate of 1.2288 Mcps by following the CDMA2000 1x standard. Then, the half-band filter is used to interpolate the pulse-shaped $I Q$ signals with a sampling rate eight times the chip rate before inserting them into the envelope calculator and subsequently into the predistorter, delay unit, and $I Q$ modulator. Before entering the delta-sigma modulator, the first component on the envelope-following path, a linear interpolator is used to further increase the sampling rate up to 32 times the chip rate. In implementation, the envelope calculator is realized based on the coordinate rotation digital computer (CORDIC) algorithm [7] for extracting the envelope from the pulse-shaped $I Q$ signals. The predistorter is a digital arithmetic unit synthesized according to the curve-fitted polynomial for compensating the distortion resulting from the nonlinear $V_{\text {in }}-V_{\text {out }}$ relation. The delay unit constructed by a stack of registers is to compensate the time delay difference between the envelope-following and quadrature-modulated signal paths. It is noted that the delay unit has been tuned to have the optimum modulation quality. The $I Q$ modulator mainly relies on a vector signal generator equipped with a two-channel digital-to-analog converter (DAC). It can output a 1.9-GHz QPSK-modulated CDMA2000 $1 \times$ signal with error vector magnitude $($ EVM) typically less than $1 \%$.

\section{EXPERIMENTAL RESULTS}

The implemented prototype transmitter is applied to generate the QPSK-modulated CDMA2000 $1 \times$ signal at the frequency of $1.9 \mathrm{GHz}$. Considering that the PAPR is about $6 \mathrm{~dB}$ for the QPSK modulation, the Class-E PA having a peak CW output power of $27 \mathrm{dBm}$ is qualified to output the standard CDMA2000 $1 \times$ signal with an average power up to about $21 \mathrm{dBm}$. The PAE for the prototype transmitter can be evaluated as

$$
\begin{aligned}
\mathrm{PAE} & =\frac{P_{\mathrm{out}}-P_{\mathrm{in}}}{P_{\mathrm{dc}}} \\
& =\frac{P_{\mathrm{out}}-P_{\mathrm{in}}}{P_{\mathrm{env}}} \cdot \frac{P_{\mathrm{env}}}{P_{\mathrm{dc}}}=\mathrm{PAE}^{\prime} \cdot \eta_{e}
\end{aligned}
$$

where $P_{\text {out }}$ and $P_{\text {in }}$ represent the average modulated power at the output and input, respectively. In (5), the PAE can be also expressed as the product of the PAE with respect to Class-E PA (denoted by $\mathrm{PAE}^{\prime}$ ) and the envelope processing efficiency. It is particularly noted that the PAEs evaluated for the Class-E PA in the digital modulation test are approximately $10 \%-20 \%$

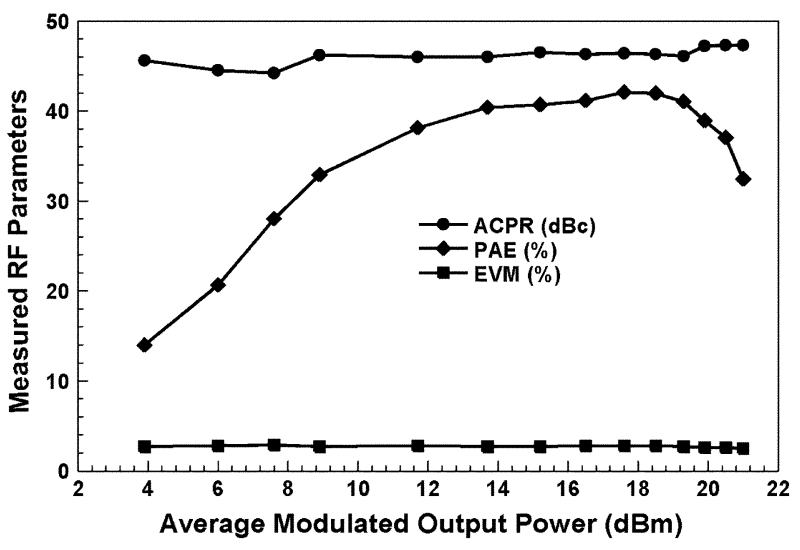

Fig. 5. Measured RF parameters for the proposed EER transmitter applied to the generation of a $1.9-\mathrm{GHz}$ CDMA2000 $1 \times$ signal.

smaller, depending on the average modulated output power, than those in the CW test.

Fig. 5 shows the measured PAE, EVM, and ACPR for the prototype transmitter. It can be seen that the prototype transmitter can achieve an almost constant EVM and ACPR value of about $2.7 \%$ and $47 \mathrm{dBc}$, respectively, over more than a decade range of average modulated output power from 4 to $21 \mathrm{dBm}$. The PAE exceeds $30 \%$ as the average modulated power is above $8 \mathrm{dBm}$, but drops quickly below that power level. This can be explained by Fig. 3(b), which displays a high descending rate for $V_{\text {out }}$ smaller than $0.8 \mathrm{~V}$ (about $8 \mathrm{dBm}$ in $\mathrm{CW}$ power). Nevertheless, the prototype transmitter still has much better overall efficiency than the conventional transmitter using a linear PA when applied to today's 3G CDMA systems with transmit power control.

\section{CONCLUSION}

A promising envelope following-based RF transmitter that uses a switching-mode PA has been presented to achieve high ACPR, low EVM, and high PAE for application to a wideband and high PAPR wireless system. The success of having such a high performance is attributed to the fact that the applied predistorted envelope and quadrature-modulated signals can effectively linearize the switching-mode PA with high average efficiency.

\section{REFERENCES}

[1] J. Staudinger, B. Gilsdorf, D. Newman, G. Norris, G. Sadowniczak, R. Sherman, T. Quach, and V. Wang, " $800 \mathrm{MHz}$ power amplifier using envelope following technique," in Proc. Radio Wireless Conf., Aug. 1999, pp. 301-304.

[2] S. Abedinpour, K. Deligoz, J. Desai, M. Figiel, and S. Kiaei, "Monolithic supply modulated RF power amplifier and DC-DC power converter IC," in IEEE MTT-S Int. Dig., Jun. 2003, vol. 1, pp. 89-92.

[3] L. R. Kahn, "Single sideband transmission by envelope elimination and restoration," in Proc. IRE'52, Jul. 1952, vol. 40, pp. 803-806.

[4] D. Rudolph, "Kahn EER technique with single-carrier digital modulations," IEEE Trans. Microw. Theory Tech., vol. 51, no. 2, pp. 548-552, Feb. 2003.

[5] D. P. Kimber and P. Gardner, "Power series analysis of the class e power amplifier," in Proc. Eur. Microw. Conf., 2004, pp. 1461-1464.

[6] J. K. Jau, Y. A. Chen, T. S. Horng, and T. L. Wu, "Optimum analytical design solution to integrated Class-E amplifiers," in Proc. IASTED WNET Conf., 2005, pp. 40-44.

[7] R. Andraka, "A survey of CORDIC algorithms for FPGA based computers," in Proc. Int. Symp. FPGA, 1998, pp. 191-200. 